\section{Relapse of acute myeloid leukemia after allogeneic stem cell transplantation is associated with gain of WT1 alterations and high mutation load}

In relapsed acute myeloid leukemia (AML), the only chance for cure is allogeneic stem cell transplantation (allo-SCT) requiring a suitable donor as well as medical fitness of the recipient. ${ }^{1}$ Although in the majority of transplanted AML patients, the donor immune system engrafts successfully, disease relapse after allo-SCT is the most common treatment failure occurring in almost half of patients. ${ }^{2}$ Relapse after allo-SCT may be caused by an immune escape of the AML cells after successful engraftment as well as by the patient's immune system bypassing the immunosuppression and thus counteracting the donor immune cells. While the genetic profile of relapsed AML after chemotherapy largely matches that of AML at initial diagnosis with rather few changes occurring during chemotherapy, ${ }^{3}$ little is known about the genetic profile of relapsed AML after allo-SCT. Both the conditioning regimen and the graft-versus-leukemia effect may alter the genetic landscape of transplanted AML. Ultimately, the molecular mechanisms leading either to graft failure or immune escape of AML cells still remain unclear. Recently, a study by Quek et al. described mutations in AML patients undergoing allo-SCT, however, it was limited to positions of known variants only and did not include a longitudinal analysis of AML patients. ${ }^{4}$ In order to unravel the clonal evolution of relapsed AML after allo-SCT, we performed exome sequencing of matched samples from $12 \mathrm{AML}$ patients, collected at multiple timepoints from initial diagnosis to relapse after alloSCT. Despite the relatively small cohort, the present study is the most comprehensive genetic analysis of AML relapse after allo-SCT carried out thus far, with exome sequencing of a total of 53 unique samples. The genetic analysis in the transplant setting is resource intensive and technically challenging, since it requires germline controls both from the donor and the recipient.

A total of seven AML patients (UPN P01-P07) were sequenced at the following five time points: initial diagnosis (Dx), complete remission after chemotherapy (CRCT), relapse after chemotherapy (Rel-CT), complete remission after allo-SCT (CR-SCT), and relapse after alloSCT (Rel-SCT) (Figure 1A). Furthermore, we selected another five patients that had already been transplanted in CR-CT and sequenced the specimen at the time points Dx, CR-CT, CR-SCT, and Rel-SCT (UPN P08-P12) (Figure 1B). Samples at CR-CT were used as germline control and available for all patients $(n=12)$, samples at CR-SCT were used to exclude donor germline variants (available for 10 patients). On average, 47.6 million reads were sequenced per sample, the mean coverage of the target region was $99.6 x$ (range $66.5 x$ to $130.1 x$ ). At least $94.4 \%$ of the target region was covered 10x or higher. Detailed sequencing metrics are summarized in Online Supplementary Table S1. Sample preparation and data analysis were performed as described previously ${ }^{5}$ with a minimum variant allele frequency (VAF) of $10 \%$ and a minimum of five supporting reads. Variants with VAF at CR-CT $<2 \%$ were called somatic, variants with VAF at CR-CT $\geq 2 \%$ were called persistent if detected in genes known to be cancer drivers. At the time point of Rel-SCT, somatic variants were detected using CR-CT and CRSCT samples as controls (Figure 1). As CR-SCT samples were not available for patients $\mathrm{P} 05$ and P08, we estimated the donor cell fraction after SCT in these two patients by VAFs of obvious donor germline variants, and subsequently excluded Rel-SCT specific variants with a VAF in
A

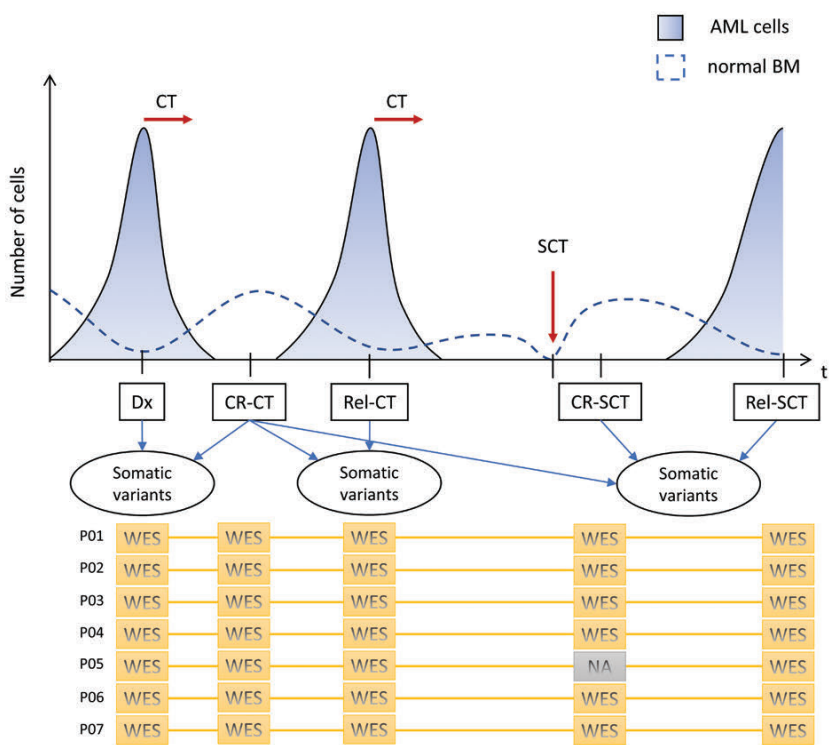

B

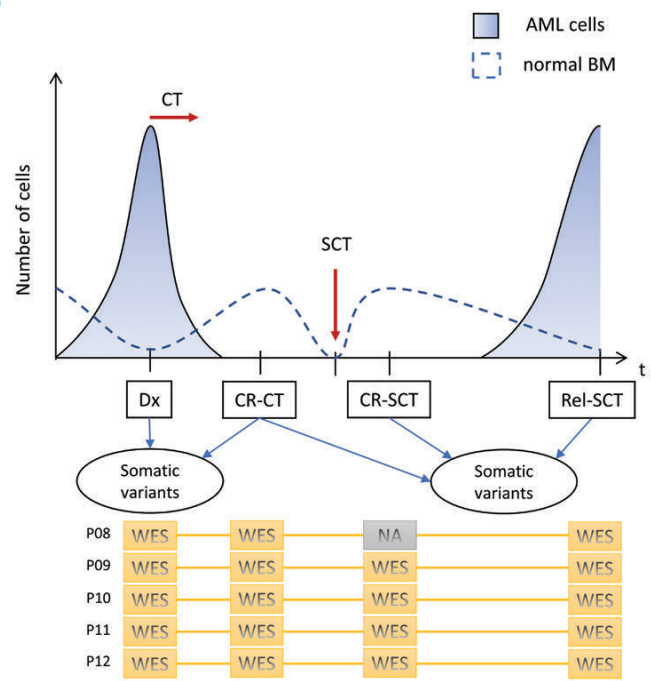

Figure 1. Schematic view of study design and the abundance of AML cells and normal bone marrow cells, respectively. Somatic variants were called with respect to the corresponding remission sample(s). Corresponding patient samples are illustrated below, yellow boxes labelled with WES represent samples that were sequenced, gray boxes represent samples that could not be sequenced. (A) Seven patients had a first relapse after chemotherapy, followed by a second CR and subsequent transplantation. (B) Five patients who were transplanted already in first CR. SCT: stem cell transplantation; CT: chemotherapy; AML: acute myeloid leukemia; CR-CT: complete remission after chemotherapy; CR-SCT: complete remission after SCT; BM: bone marrow; Dx: diagnosis; Rel-CT: relapse after chemotherapy; Rel-SCT: relapse after stem cell transplantation. 

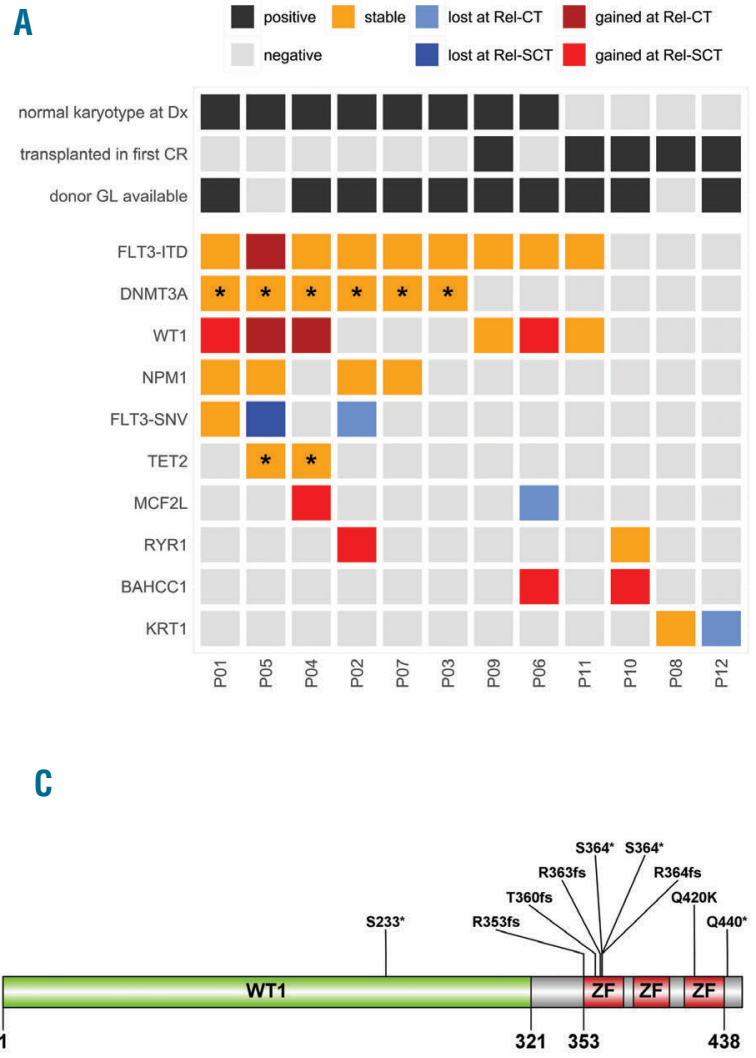

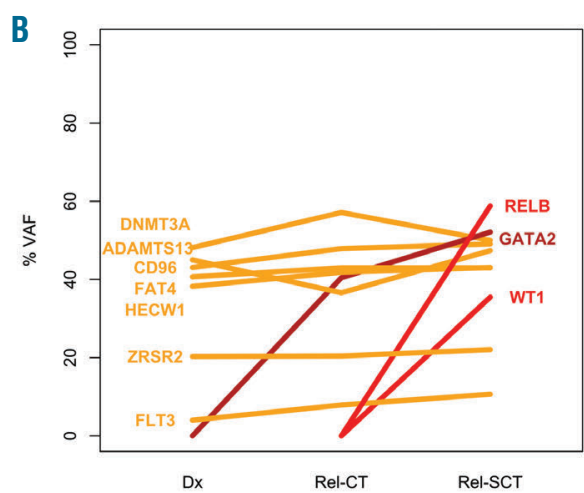

D

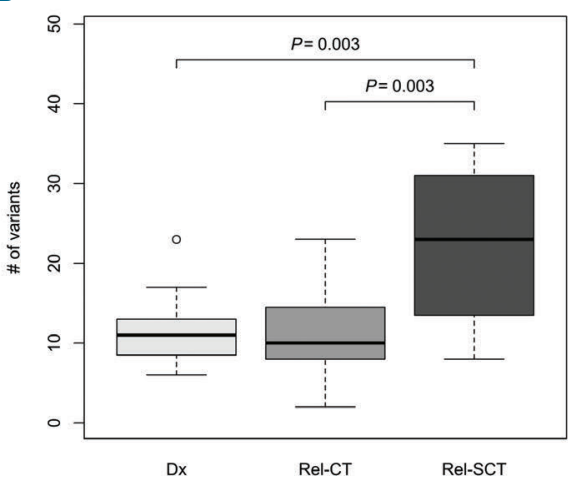

Figure 2. Somatic mutations in AML relapse after allogeneic SCT. (A) Recurrent mutations detected in patients, each column represents one patient. Each row represents the status of a gene or property indicated on the left. The existence of variants at an individual time point is indicated using the color code at the top. Persistent variants detectable at CR-CT samples are marked with an asterisk (*). (B) Exemplary clonal evolution of UPN P01. Individual mutations are displayed by a line connecting VAFs at indicated time points, colored according to their evolutionary pattern. Orange: stable mutation; dark red: mutation gained at Rel-CT and stable at Rel-SCT; light red: mutation gained at Rel-SCT. (C) Domain graph of mutations in WT1 (NM_000378.4). Amino acid positions and protein changes are presented at the top, domain positions are shown at the bottom. The graph was generated using IBS software, with domain information from PFAM database. An asterisk (*) indicates an introduced stop codon; fs indicates a frame shift. (D) Mutation load at individual time points. Dx: diagnosis; CR: complete remission; GL: germline control sample. Rel-CT: relapse after chemotherapy; Rel-SCT: relapse after stem cell transplantation; VAF: variant allele frequency; ZF: zinc finger.

the range of putative donor germline variants (Online Supplementary Figure S1). The criteria for donor germline variants were defined as: (I) presence at Rel-SCT only (VAF $=0 \%$ in all previous samples), (II) annotation as common single nucleotide polymorphisms (SNPs) in the $\mathrm{dbSNP}$ database (minor allele frequency $(\mathrm{MAF}) \geq 1 \%$ ), and (III) minimum coverage of $100 \mathrm{x}$ in order to increase the reliability of cell fraction estimation by VAFs. Thereby, we detected $>500$ donor germline variants per sample (Online Supplementary Figure S1A). Rel-SCT specific variants not matching these criteria but with a VAF in the range of donor germline variants were excluded from somatic variant analysis in the two patients without an appropriate control (P05: 10\%-26.7\%, P08: 25.5\%$100 \%$ ) (Online Supplementary Figure S1B). While somatic variants in leukemia subclones might be missed, this approach dramatically reduces the false positive rate, as donor germline variants cannot be confounded with RelSCT specific somatic variants. Online Supplementary Figure $S 1 C, D$ show the robustness of this approach, as we applied our method to CR-SCT samples with a known donor cell fraction of $100 \%$. Finally, all somatic variants underwent exclusion of sequencing artifacts by an inhouse blacklist of error-prone regions and manual alignment inspection in order to reduce false positive variant calls. FLT3-ITD and NPM1 mutation status was obtained from routine assays.

The median age of the patients was 47.5 (range 21-73), most patients (8/12) were cytogenetically normal (CN) at Dx (Figure 2A, Online Supplementary Table S2). Aberrant cytogenetics at Dx included $t(9 ; 11)(n=1)$, trisomy $8(n=1)$ and complex karyotypes $(n=2)$. In our cohort, a median of 26 somatic variants were detected by next-generation sequencing at any time point per patient (range 10-39) with a total of nine genes recurrently mutated (Figure 2A, Online Supplementary Figure S2, Online Supplementary Table S3). Consistent with a high risk of relapse, FLT3ITD was the most common mutation $(n=9)$, followed by mutations in DNMT3A, WT1, and NPM1 $(\mathrm{n}=6, \mathrm{n}=6, \mathrm{n}=4$, respectively). Mutations in DNMT3A and NPM1 were stable at all time points with $6 / 6$ DNMT3A mutations showing persistence at CR-CT. Only two patients had mutations in WT1 at Dx, corresponding to the previously reported overall prevalence of WT1 mutations in diagnostic AML samples of $6-13 \%{ }^{3,6,7}$ Interestingly, WT1 mutations were recurrently gained during the course of the disease $(n=4)$ resulting in a substantially higher number of WT1 mutations at Rel-SCT (6/12 patients). In order to determine if gained WT1 mutations were acquired de novo during therapy, or already present at subclonal levels 
at earlier time points, we evaluated targeted amplicon sequencing data with a minimum coverage of $595 \mathrm{x}$ at WT1 mutation positions. We identified two subliminal mutations in only one out of four patients with gained WT1 mutations (VAF $<1 \%$ each) at relapse prior to SCT, suggesting a subclone harboring both mutations (UPN P06, p.Q420K and p.S364*). Recurrent mutation gains were also detected in BAHCC1 and RYR1 ( $\mathrm{n}=2$ each), whereas a recurrent loss was observed only for FLT3 point mutations, both located in the C-terminal tyrosine kinase domain (D835K, n=2), while another FLT3 point mutation (F590N, UPN P01) was stable throughout the follow-up. In general, the majority of mutations detected at Dx were also found at Rel-CT and Rel-SCT (120/137, $88 \%$ ), however, individual mutations were lost or gained during the course of the disease (Figure 2B, Online Supplementary Figure S2 and Figure S3). Of note, all WT1 mutated patients harbor at least one mutation in the hotspot region of exon 7 , introducing a premature stop codon (Figure 2C, Online Supplementary Table S3). Overall, the number of somatic variants was similar at Dx (median 11, range 6-23) and Rel-CT (median 10, range 2-23), while after allo-SCT, the mutation load was significantly higher (median 23, range 8-35, $P=0.003$, two-tailed unpaired Student's $t$-test, Figure 2D, Online Supplementary Figure S2). Although we estimated the donor cell fraction and excluded variants likely representing donor germline variants, both patients without CRSCT samples were excluded from the mutational load analysis, as the genetic burden of Rel-SCT might be underestimated in these patients. In general, the VAF of most Rel-SCT specific mutations suggest that a major clone is harboring these alterations (Online Supplementary Figure S3, Online Supplementary Table S3).

During the course of AML, patients show a substantial number of stable mutations at multiple time points. Only few changes of the mutational profile can be observed between Dx and Rel-CT. Individual gain or loss of mutations points towards relapse originating from either the dominant AML clone at Dx with few additional alterations, or from a very similar but ancestral subclone present at Dx expanding after chemotherapy. In general, resistance to chemotherapy might be mediated even by a single mutation or only a few genetic alterations which can be found after CR-CT. In a previous study, we showed that WT1 mutations were gained in relapsed CNAML patients after CR-CT $(14 \%$ at Rel-CT vs. $8 \%$ at $\mathrm{Dx}){ }^{3}$ In contrast, after allo-SCT the mutational load in $\mathrm{AML}$ is significantly higher with frequent acquisition of WT1 loss-of-function mutations constituting a potential mechanism of escape from the donor immune system. The high overall mutational load observed at Rel-SCT might be related to the mutagenic effects of conditioning regimens, including both chemotherapy and total body irradiation or an increased need for additional alterations in order to escape the therapy. The observed association of WT1 mutations with both Rel-CT and Rel-SCT in the present study is consistent with results presented at the recent EHA meeting. ${ }^{8}$ Previous studies have shown that WT1 mutations are associated with chemotherapy resistance in $\mathrm{CN}^{-\mathrm{AML}^{9}}$ and gained at relapse after chemotherapy. ${ }^{10}$ The study by Quek et al. reports a total of five WT1 mutations at $\mathrm{Dx}$ in 49 relapsing patients $(10 \%)$. Moreover, the authors analyzed paired pre- and postSCT samples of 29 patients and observed a total of three WT1 mutations in AML after allo-SCT. ${ }^{4}$ Thus, the prevalence of WT1 mutations after allo-SCT is strikingly higher in our study (6/12 vs. 3/29, 50\% vs. $10 \%)$. Further, one of the WT1 mutations detected at Rel-SCT in that study was detected only at a subclonal level with a VAF of $7 \%$, which is below our detection cutoff. Quek and colleagues might have missed additional WT1 mutations as they did not include germline samples in order to detect somatic variants systematically. Instead, they focused on previously reported somatic variants in hematopoietic diseases and considered novel variants only if adjacent to known positions. Moreover, the authors reported only mutations found by two different variant callers, namely GATK and VarScan. Thus, the burden of WT1 mutations might have been underestimated in that study. In distinct genetic subgroups of AML, WT1 mutations have been described with varying impact on prognosis. ${ }^{11,12}$ In biallelic CEBPA mutant AML, the prognostic relevance remains controversial, while in CN-AML WT1 mutations associate with shorter event-free survival. WT1 mutations have been described as rare events in CN-AML and in our cohort, only one out of eight CN-AML patients was WT1-mutated at Dx. At Rel-SCT, all WT1 mutation gains were detected in CN-AML patients. Thus, a total of 5/6 WT1 mutations were detected in $\mathrm{CN}-\mathrm{AML}$ patients, pointing towards the aggressiveness of WT1-mutated disease in this genetic subgroup. As we used samples at CR-CT as germline control samples, our study may be enriched for CN-AML patients $(8 / 12)$ who are more likely to achieve a CR. In contrast to WT1 gene expression as a prognostic marker in pre-allo-SCT AML, ${ }^{13,14}$ little is known about the prognostic value of WT1 mutations in patients allografted for AML. Quek et al. reported that the presence of WT1 mutations before allo-SCT correlates with increased relapse risk. ${ }^{4}$ Of note, in acute lymphoblastic leukemia WT1 mediates the graft-versus-leukemia effect after alloSCT. ${ }^{15}$ It is intriguing to speculate that the truncating mutations of WT1 may disrupt a critical epitope of AML cells which is targeted by the donor immune system. Recurring BAHCC1 or RYR1 mutations have not been described in AML before, but recurrent deletions of BAHCC1 in hepatocellular carcinoma point towards a potential role of this gene in cancer, ${ }^{16}$ while $R Y R 1$ was shown to be expressed in a variety of hematopoietic cells ${ }^{17}$ and to be associated with immune response and T-cell activation. ${ }^{18,19}$

Taken together, deciphering the evolution of AML relapse after allo-SCT and the potential role of WT1 mutations in disease progression are essential to resolve this challenging clinical situation.

Sebastian Vosberg, ${ }^{1,2,3,4}$ Luise Hartmann ${ }^{1,2,3,4}$
Klaus H. Metzeler,,$^{1,2,3,4}$ Nikola P. Konstandin,
Stephanie Schneider, ${ }^{4,5}$ Ashok Varadharajan,
Andreas Hauser, ${ }^{6}$ Stefan Krebs, ${ }^{6}$ Helmut Blum,
Stefan K. Bohlander, ${ }^{7}$ Wolfgang Hiddemann, ${ }^{1,2,3,4}$
Johanna Tischer, ${ }^{8}$ Karsten Spiekermann ${ }^{1,2,3,4}$
and Philipp A. Greif ${ }^{1,2,3,4}$

Experimental Leukemia and Lymphoma Research, Department of Medicine III, University Hospital, LMU Munich, Germany; ${ }^{2}$ German Cancer Consortium (DKTK), partner site Munich, Germany; ${ }^{3}$ German Cancer Research Center (DKFZ), Heidelberg, Germany; ${ }^{4}$ Laboratory for Leukemia Diagnostics, Department of Medicine III, University Hospital, LMU Munich, Germany; Institute of Human Genetics, University Hospital, LMU Munich, Germany; 'Laboratory for Functional Genome Analysis, Gene Center, LMU Munich, Germany; ${ }^{7}$ Leukaemia and Blood Cancer Research Unit, Department of Molecular Medicine and Pathology, The University of Auckland, New Zealand and ${ }^{8}$ Hematopoietic Stem Cell Transplantation, Department of Medicine III, University Hospital, LMU Munich, Germany

Funding: This study was supported by the German Research Foundation (DFG), Collaborative Research Centre (SFB) 1243 
'Cancer Evolution', projects A06, A07, A08 and Z2). SKB is supported by Blood Cancer New Zealand and the family of Marijanna Kumerich.

Correspondence:pgrei@med.uni-muenchen.de doi:10.3324/haematol.2018.193102

Information on authorship, contributions, and financial \& other disclosures was provided by the authors and is available with the online version of this article at wWw. haematologica.org.

\section{References}

1. Gupta V, Tallman MS, Weisdorf DJ. Allogeneic hematopoietic cell transplantation for adults with acute myeloid leukemia: myths, controversies, and unknowns. Blood. 2011;117(8):2307-2318.

2. Barrett AJ, Battiwalla M. Relapse after allogeneic stem cell transplantation. Expert Rev Hematol. 2010;3(4):429-441.

3. Greif PA, Hartmann L, Vosberg S, et al. Evolution of cytogenetically normal acute myeloid leukemia during therapy and relapse: An exome sequencing study of 50 patients. Clin Cancer Res. 2018; 24(7):1716-1726

4. Quek L, Ferguson P, Metzner M, et al. Mutational analysis of disease relapse in patients allografted for acute myeloid leukemia. Blood Adv. 2016;1(3):193-204.

5. Herold T, Metzeler KH, Vosberg S, et al. Isolated trisomy 13 defines a homogeneous AML subgroup with high frequency of mutations in spliceosome genes and poor prognosis. Blood. 2014;124(8):13041311.

6. Cancer Genome Atlas Research N. Genomic and epigenomic landscapes of adult de novo acute myeloid leukemia. N Engl J Med. 2013;368(22):2059-2074.

7. Metzeler KH, Herold T, Rothenberg-Thurley M, et al. Spectrum and prognostic relevance of driver gene mutations in acute myeloid leukemia. Blood. 2016;128(5):686-698.

8. Sala E, Biavasco F, Bucci G, et al. Profiling the mutational landscape of acute myeloid leukemia at relapse after chemotherapy and allogeneic hematopoietic stem cell transplantation. Haematologica. 2017;102(S2):208-209.

9. Virappane P, Gale R, Hills R, et al. Mutation of the Wilms' tumor 1 gene is a poor prognostic factor associated with chemotherapy resistance in normal karyotype acute myeloid leukemia: the United
Kingdom Medical Research Council Adult Leukaemia Working Party. J Clin Oncol. 2008;26(33):5429-5435.

10. Hou HA, Huang TC, Lin LI, et al. WT1 mutation in 470 adult patients with acute myeloid leukemia: stability during disease evolution and implication of its incorporation into a survival scoring system. Blood. 2010;115(25):5222-5231

11. Krauth MT, Alpermann T, Bacher U, et al. WT1 mutations are secondary events in AML, show varying frequencies and impact on prognosis between genetic subgroups. Leukemia. 2015;29(3):660667.

12. Tien FM, Hou HA, Tang JL, et al. Concomitant WT1 mutations predict poor prognosis in acute myeloid leukemia patients with double mutant CEBPA. Haematologica. 2018 May 17. [Epub ahead of print]

13. Candoni A, De Marchi F, Zannier ME, et al. High prognostic value of pre-allogeneic stem cell transplantation minimal residual disease detection by WT1 gene expression in AML transplanted in cytologic complete remission. Leuk Res. 2017;63:22-27.

14. Nomdedeu JF, Esquirol A, Carricondo M, et al. Bone marrow WT1 levels in allogeneic hematopoietic stem cell transplantation for acute myelogenous leukemia and myelodysplasia: clinically relevant time points and 100 copies threshold value. Biol Blood Marrow Transplant. 2018;24(1):55-63.

15. Rezvani K, Yong AS, Savani BN, et al. Graft-versus-leukemia effects associated with detectable Wilms tumor-1 specific T lymphocytes after allogeneic stem-cell transplantation for acute lymphoblastic leukemia. Blood. 2007;110(6):1924-1932.

16. Nalesnik MA, Tseng G, Ding Y, et al. Gene deletions and amplifications in human hepatocellular carcinomas: correlation with hepatocyte growth regulation. Am J Pathol. 2012;180(4):1495-1508.

17. Clark AJ, Petty HR. Differential intracellular distributions of inositol trisphosphate and ryanodine receptors within and among hematopoietic cells. J Histochem Cytochem. 2005;53(7):913-916.

18. Vukcevic M, Zorzato F, Keck S, et al. Gain of function in the immune system caused by a ryanodine receptor 1 mutation. J Cell Sci. 2013;126(Pt 15):3485-3492.

19. Wolf IM, Diercks BP, Gattkowski E, et al. Frontrunners of T cell activation: Initial, localized Ca2+ signals mediated by NAADP and the type 1 ryanodine receptor. Sci Signal. 2015;8(398):ra102. 